\title{
REVIEW
}

\section{Mitogen activated protein (MAP) kinase signal transduction pathways and novel anti-inflammatory targets}

\section{W Hommes, M P Peppelenbosch, S J H van Deventer}

Gut 2003;52:144-151

Over the last decade important advances have been made in our understanding of the molecular events underlying cellular responses to extracellular signals. Increased understanding of signal transduction mechanisms and gene regulation involved in immune responses has created opportunities for the discovery of novel therapeutic compounds useful in treating inflammatory disorders. One of the best studied signalling routes is the mitogen activated protein (MAP) kinase signal transduction pathway which plays a crucial role in many aspects of immune mediated inflammatory responses. Here, our current understanding of the MAP kinase pathway is reviewed, as well as recent advances in the design of novel agents that are able to modulate the activity of these signalling cascades.

See end of article for authors' affiliations

Dr DW Hommes

Department of Gastroenterology and Hepatology, Academic Medical Centre, C2-1 16 , Meibergdreef 9, 1105 AZ Amsterdam, the

Netherlands:

d.w.hommes@amc.uva.nl

Accepted for publication 3 September 2002

\begin{abstract}
SUMMARY
Proper regulation of genes in all forms of cellular life is dependent on intracellular regulatory circuits or signal transduction pathways. Among eukaryotic cells, phosphorylation of intracellular factors followed by specific gene transcription is a universal outcome of such signal transduction pathways, and the major elements of such pathways are similar in species as different as humans, fungi, and plants. In mammalian species, these ancient regulatory circuits maintain the balanced gene transcription necessary for correct cell growth, differentiation, and death. Scientific interest in these information highways from the cell surface to the nucleus has exploded over the past years, and understanding of the biology of these signalling cascades has progressed dramatically. One of the best studied signalling routes is the mitogen activated protein (MAP) kinase signal transduction pathway which plays a crucial role in many aspects of immune mediated inflammatory responses. Hence members of this family of kinases have come to be appreciated as key cellular signal transducers and attractive targets for drug development. This has led to current initiation of clinical trials in inflammatory disease states evaluating small molecule inhibitors of MAP kinase proteins and encouraging results have been obtained. Because it is anticipated that several small MAP kinase inhibiting molecules will be evaluated for efficacy in inflammatory diseases, here we review current knowledge of the MAP kinase signalling pathways as well as potential inhibitory drugs.
\end{abstract}

\section{INTRODUCTION}

During the last decade important advances have been made in our understanding of the molecular events underlying cellular responses to extracellular signals. This scientific progress has set the stage for an exciting new field of research: pharmacological modulation of signal transduction pathways to control gene expression. Altered gene expression plays a key role in the pathogenesis of many inflammatory diseases such as pancreatitis, rheumatoid arthritis, hepatitis, psoriasis, and inflammatory bowel disease. It is only in recent years that signal transduction mechanisms responsible for inducing inflammatory gene expression have been identified. These mechanisms seem fundamental in the initiation of inflammatory responses. Products of induced inflammatory genes include cytokines, chemokines, and adhesion molecules that serve to promote recruitment of immunocompetent cells from the circulation to the affected site which results in an inflammatory injury. It is for this reason that these intracellular pathways have come to be appreciated as attractive targets for drug development.

The aim of this review is to present the current understanding of the MAP kinase pathway, a key mechanism of inflammatory signal transduction in eukaryotic cells. Furthermore, we will review recent advances in the design of novel agents that are able to modulate the activity of these signalling cascades.

\section{SIGNAL TRANSDUCTION AND CONTROL OF GENE EXPRESSION.}

There are two major routes from extracellular signalling proteins to changes in gene transcription (fig 1). Firstly, several intracellular self propagating phosphorylation cascades have been identified that activate different resident nuclear transcription factors. Secondly, a number of latent cytoplasmic transcription factors are activated after cell surface receptor-ligand interactions that

Abbreviations: AP-1, activating protein 1; ATF-2, activating transcription factor 2; ERK, extracellular signal regulated kinase; IFN, interferon; IL, interleukin; JAK, Janus kinase; JNK, c-Jun $\mathrm{NH}_{2}$ terminal kinase; LPS,

lipopolysaccharide; MAP, mitogen activated protein; MAPK, MAP kinase; MAPKAP, MAP kinase activated protein kinase; MAPKK, MAP kinase kinase; MAPKKK, MAP kinase kinase kinase; MEF, myocyte enhance factor; MEK, MAP/ERK kinase; MKK, MAP kinase kinase; NF, nuclear factor; STAT, signal transducers and activators of transcription; TGF, transforming growth factor; TNF, tumour necrosis factor. 


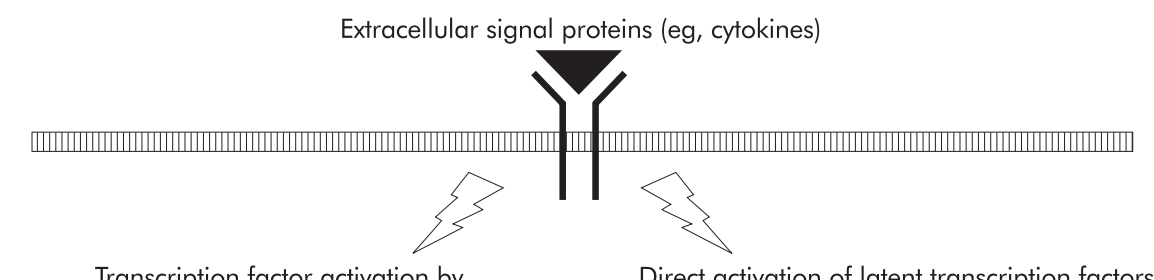
phoshorylation cascades

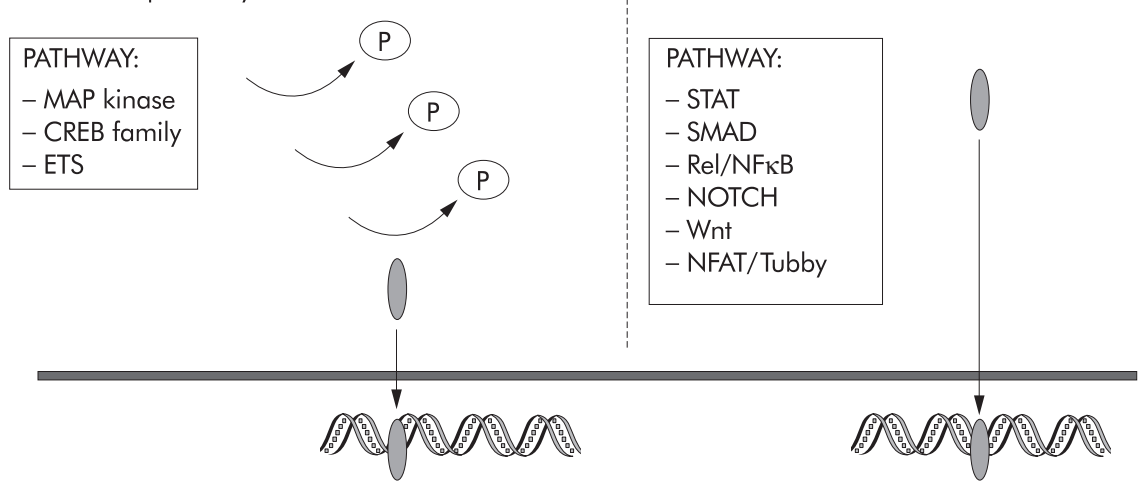

Figure 1 Different routes from extracellular signalling proteins to changes in gene transcription. Left: Intracellular phosphorylation cascades activate nuclear transcription factors. Right: Latent cytoplasmic transcription factors are activated on receptor-ligand interaction.

accumulate in the nucleus to drive transcription. Recently, a classification system for transcription factors was proposed to structure this still increasing repertoire of intracellular proteins regulating cell development and cell specialisation. ${ }^{1}$

MAP kinases, which belong to a large family of serine/ threonine kinases, constitute major inflammatory signalling highways from the cell surface to the nucleus. ${ }^{2}$ Other kinase cascades include those that are activated after increases in intracellular second messengers (including cAMP and $\mathrm{Ca}^{2+}$ ) and ligand interaction with $G$ coupled cell surface receptor proteins and receptor tyrosine kinases. ${ }^{3}$ Usually such pathways result in the phosphorylation and subsequent activation of transcription factors, producing altered gene transcription.

Eight principal pathways for activation of latent cytoplasmic transcription factors are now recognised. The two pathways that are directly activated at the cell surface receptor are the family of SMAD molecules that are involved in signal transduction of the transforming growth factor $\beta$ (TGF- $\beta$ ) superfamily of ligands, ${ }^{4}$ and the STAT family ( signal transducers and activators of transcription) which are activated by more than 20 cytokines sharing Janus kinase (JAK) associated receptors that phosphorylate the respective receptors on tyrosine residues to initiate signal transduction. ${ }^{5}$ In short, inactive JAK enzymes are loosely attached to the cytoplasmic domains of specific cytokine receptors (including interferon (IFN)- $\alpha / \beta / \gamma$, interleukin (IL)-2, IL-4, IL-6, IL-10, and IL-12). After ligand binding, the receptor associated JAKs become active through phosphorylation, and in their turn phosphorylate tyrosine residues in the cytoplasmic portions of the clustered receptors. Subsequently, cytosolic STAT proteins recognise these phosphotyrosine moieties and are phosphorylated after attachment to the receptors by receptor associated JAKs. After binding of two STAT proteins, they dissociate from the receptor and migrate to the nucleus where they bind to DNA sequences in the promoter regions of cytokine responsive genes and activate gene transcription. STAT activation may have important consequences. For instance, in $\mathrm{T}$ helper cell differentiation, STAT4 activation is sufficient to confer a T helper 1 cell phenotype whereas STAT6 activation causes transition from a precursor cell to a T helper 2 cell.

"The Wnt signalling pathway is pivotal in normal and malignant development"
Four other important pathways that can activate latent transcription factors require cytoplasmic serine phosphorylation and/or proteolysis. This group includes the Rel/nuclear factor $\kappa \mathrm{B}(\mathrm{NF \kappa B})$ family which is activated by, and essential for, correct response to a wide variety of extracellular stimuli such as tumour necrosis factor $\alpha$ (TNF- $\alpha$ ), IL-1, and viral and bacterial products. ${ }^{6}$ This group also includes the Wnt pathway and the Notch pathway. The Wnt signalling pathway is pivotal in normal and malignant development. For example, adenomatous polyposis coli protein has been thought to function as a tumour suppressor through its involvement in the Wnt/ $\beta$ catenin signalling pathway. ${ }^{7}$ The Notch signalling pathway has been implicated in lymphopoiesis: recent data suggest that Notch activity plays a pivotal role in T cell lineage commitment from the common lymphoid progenitor. ${ }^{8}$ Furthermore, Notch signalling seems crucial in the developing nervous system; its function determines cell fate from the first segregation of neuronal precursors to the terminal specification of cells as neurones and glia. ${ }^{10}$

Finally, fluctuations in cytoplasmic second messengers $\left(\mathrm{Ca}^{2+}\right.$ and phosphoinositide concentrations) can activate latent cytoplasmic transcription factors such as nuclear factors in activated T cells (NFAT) ${ }^{11}$ and Tubby, a product of a gene which is associated with obesity. ${ }^{12}$ A detailed analysis of the aforementioned signalling mechanisms lies beyond the aim of this review, and we will focus on the most ancient and conserved signalling pathway that is pivotal during immune responses: the MAP kinase pathway.

\section{MAP KINASE PATHWAY}

MAP kinases are an evolutionarily conserved family of enzymes that form a highly integrated network required to achieve specialised cell functions controlling cell differentiation, cell proliferation, and cell death. ${ }^{13}$ These cytoplasmic proteins can modulate the activities of other intracellular proteins by adding phosphate groups to their serine/threonine amino acids (fig 2). To date, over 20 MAP kinase isoforms have been reported, which are summarised in table 1 . Activation of MAP kinase enzymes themselves is unusual in that they require phosphorylation on both a threonine (Thr) and tyrosine (Tyr) residue and thus need the activity of dual specificity kinases which are known as MAP/ERK kinases (MEKs) or MAP 


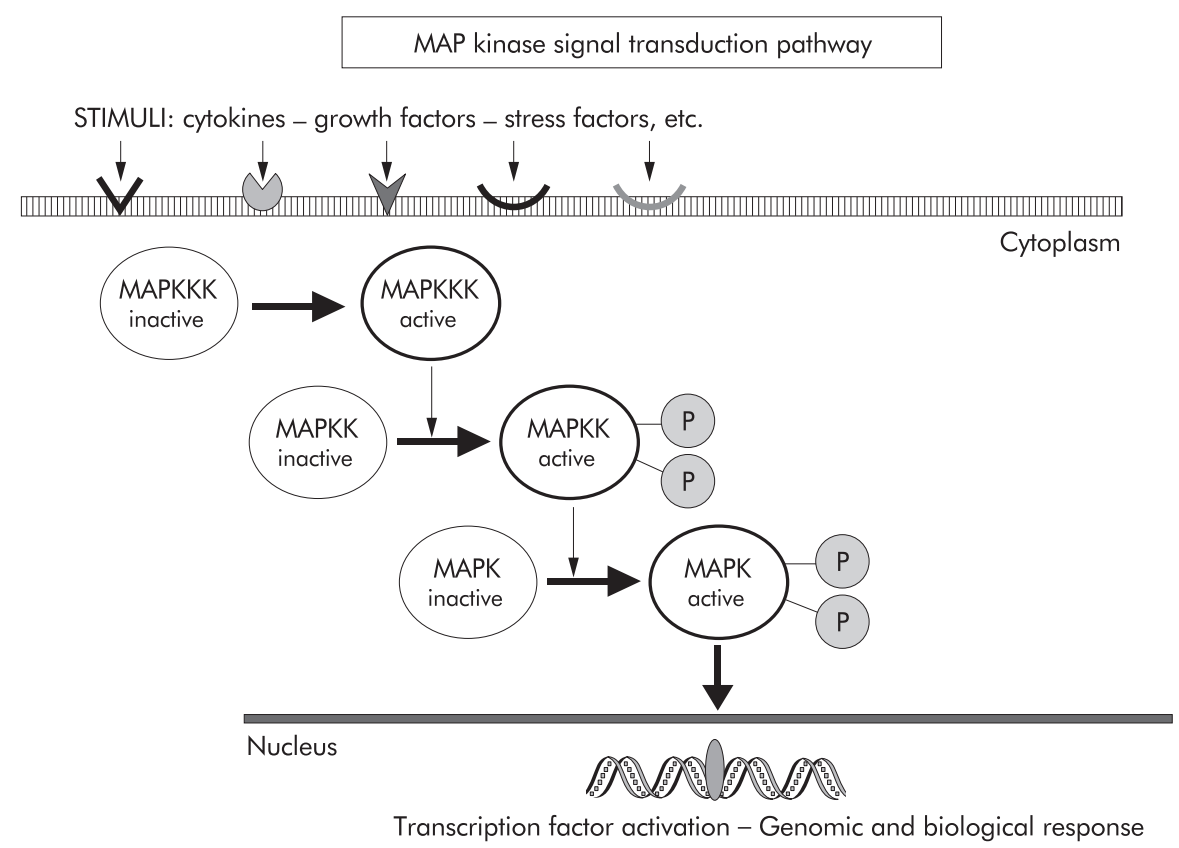

Figure 2 Different extracellular stimuli can activate the family of mitogen activated protein (MAP) kinases after receptor-ligand interactions. Members of this family activate each other by adding phosphate groups to serine/threonine amino acids. MAPK, MAP kinase; MAPKK, MAP kinase kinase; MAPKKK, MAP kinase kinase kinase.

kinase kinases (the phosphorylating potential of all other mammalian kinase families is restricted to either phosphorylation of serine/threonine residues or tyrosine residues). The specificity of MEK for MAP kinase is context dependent: all MEK enzymes exert their dual phosphorylation on Thr-XxxTyr amino acid sequence motifs but the $\mathrm{X}$ confers specificity. The amino acid $\mathrm{X}$ is glutamic acid (Glu) for extracellular regulated protein kinase (ERK), proline (Pro) for c-Jun $\mathrm{NH}_{2}$ terminal kinase (JNK), and glycine (Gly) for p38 MAP kinase. ERK MAP kinases are activated by MAP kinase kinase (MKK) I and MKK2, JNK by MKK4 and MKK7, and p38 MAP kinase by MKK3, MKK4, and MKK6 (fig 3). Hence activation of a specific MEK will result in a defined set of further cellular consequences.

The activity of MEKs is in turn controlled by phosphorylation, phosphorylated MEKs being enzymatically active. This phosphorylation status is under the critical control of so-called MAP kinase kinase kinases (MAPKKK, MKKK, or
MEKK), a family of proteins of which the c-Raf protooncogene is the most prominent member. The control of this family of enzymes and their substrate specificity is still only partially understood.

On activation of MAP kinases, transcription factors present in the cytoplasm or nucleus are phosphorylated and activated, leading to expression of certain target genes resulting in a biological response. The multiple interactions between the different MAP kinase cascades serve to integrate responses and to moderate outputs. Indeed, it has been demonstrated that MAP kinases have overlapping substrate specificities and phosphorylation of regulatory sites is shared among multiple protein kinases. ${ }^{14-16}$

As noted above, three major groups of distinctly regulated groups of MAP kinase cascades are known in humans that lead to altered gene expression: ERKl/2, JNK, and p38 MAP kinase.

\begin{tabular}{|c|c|c|c|}
\hline $\begin{array}{l}\text { MAP kinase } \\
\text { Isoform }\end{array}$ & Alternative name & Substrate & Phenotypes of MAP kinase knockout mice. \\
\hline ERK 1 & p44 MAPK & MAPKAP-K1, MNKs, MSKs, Elk1 & Defective T cell development \\
\hline ERK 2 & p42 MAPK & MAPKAP-K1, MNKs, MSKs, Elk 1 & \\
\hline ERK $3 \alpha$ & p63 MAPK & MNKs, MSKs & \\
\hline ERK $3 \beta$ & human ERK3 & MNKs, MSKs & \\
\hline ERK 4 & ERK $1 b$ & MNKs, MSKs & \\
\hline ERK 5 & & MNKs, MSKs & \\
\hline ERK 7 & & MNKs, MSKs & \\
\hline JNK 1 & SAPK $\gamma$ & c-Jun, JunD, ATF-2, Elk 1 & Defective T cell differentiation to Th2 cells \\
\hline JNK 2 & SAPK $\alpha$ & c-Jun, JunD, ATF-2, Elk1 & Defective $T$ cell differentiation to Th 1 cells \\
\hline JNK 3 & SAPK $\beta$ & c-Jun, JunD, ATF-2, Elk 1 & Resistance to excitotoxic neuronal cell death \\
\hline p38 $\alpha$ & CSBP, SAPK2 MPK2, RK, Mxi2 & MAPKAP-K2/3, MSKs, ATF-2, Elk1, MEF2c & Placental defect, insufficient production of erythropoietin \\
\hline p38 $\beta$ & $\mathrm{p} 38-2, \mathrm{p} 38 \beta_{2}$ & MAPKAP-K2/3, MSKs, ATF-2 & \\
\hline p38r & ERK6, SAPK3 & ATF2 & \\
\hline p38ס & SAPK4 & ATF2 & \\
\hline
\end{tabular}

ATF-2, activating transcription factor 2; ERK, extracellular signal regulated kinase; JNK, c-Jun $\mathrm{NH}_{2}$ terminal kinase; MAP, mitogen activated protein; MAPK, MAP kinase; MAPKAP, MAP kinase activated protein kinase; MEF, myocyte enhance factor; SAPK, stress activated protein kinase; MNKs, MARK activating protein kinases; MSKs, mitogen and stress activated protein kinases; CSBP, cytokine suppressive anti-inflammatory binding protein; RK, reactivating kinase. 


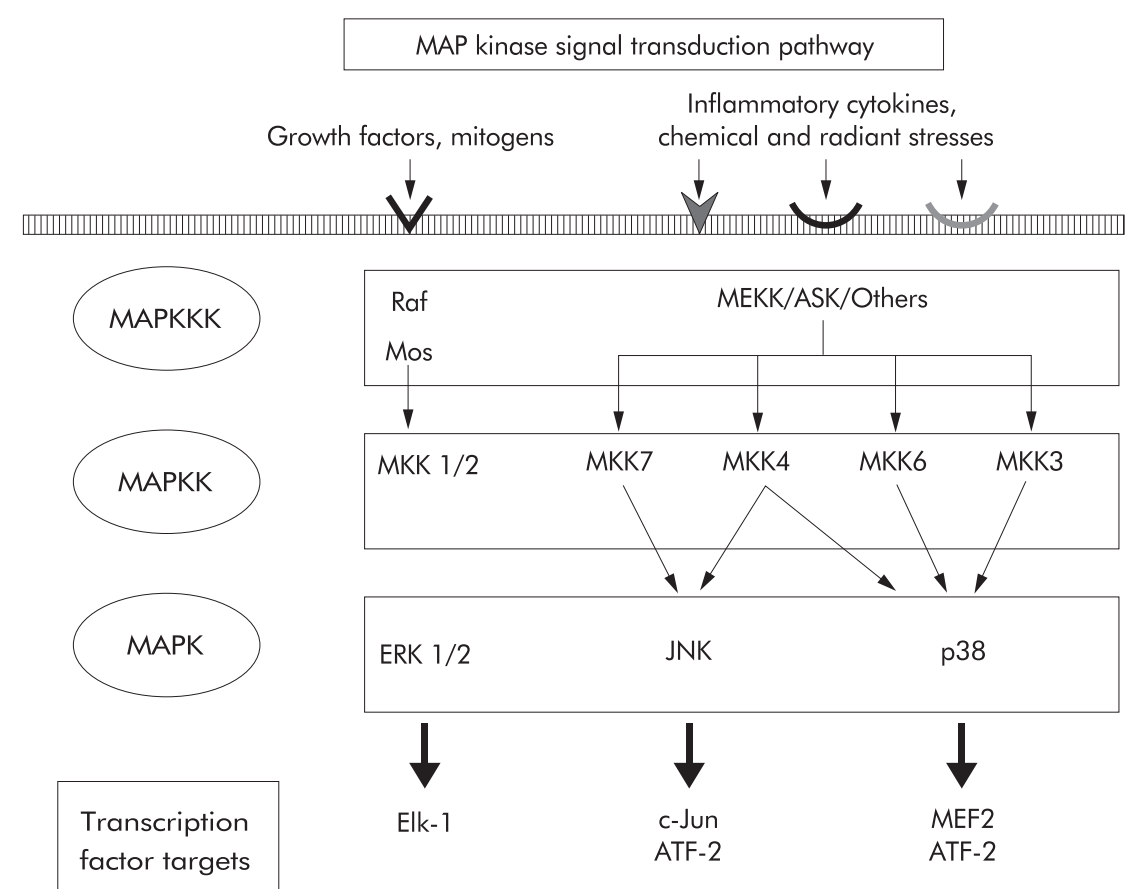

Figure 3 Result of phosphorylation of various mitogen activated protein (MAP) kinase isoforms is activation of the three main MAP kinases: ERK, JNK, and p38 MAP kinase, which have different transcription targets. ATF-2, activating transcription factor 2; ERK, extracellular signal regulated kinase; JNK, c-Jun $\mathrm{NH}_{2}$ terminal kinase; MAPK, MAP kinase; MAPKK, MAP kinase kinase; MAPKKK, MAP kinase kinase kinase; MEF, myocyte enhance factor; MEK, MAP/ERK kinase; MKK, MAP kinase kinase; ASK, apoptosis signal regulating kinase.

\section{The ERK1/2 signal transduction pathway}

The ERK signalling module was the first MAP kinase cascade to be characterised, being a vital mediator of a number of cellular fates including growth, proliferation, and survival. There are two ERK isoforms that are ubiquitously expressed, ERK1 and ERK2, and these are often referred to as p42/p44 MAP kinases. ${ }^{17}{ }^{18}$ In this cascade, MEK1 and MEK2 function as upstream MAPKK and the Raf proteins as MAPKKK (fig 3). Duration of ERK1/2 activation depends on regulating mechanisms: removal of one or both phosphates by tyrosine, serine/ threonine, or phosphatases radically decreases this MAP kinase activity. ${ }^{19}$ Furthermore, the specificity of these phosphatases is dependent on their intracellular localisation. ERKl/2 as well as other MAP kinases target not only transcription factors but also membrane proteins (that is, phospholipase $\mathrm{A}_{2}$ ) and cytoplasmic proteins (that is, downstream kinases). Results from gene knockout experiments have illustrated the importance of the ERKl/2 pathway. ${ }^{20}$ Disruption of one of the three Raf proteins known to activate ERKl/2 is invariably fatal in mice. Also, when MEKl was genetically targeted, embryonic death was observed with signs of tissue necrosis. Precisely how ERKl/2 affects cellular physiology in vivo is poorly understood. Most often an important role is attributed to ERK1/2 dependent regulation of the activating protein 1 (AP-1) family of transcription factors. Members of this family that are phosphorylated by ERK1/2 include c-Jun, c-Fos, and activating transcription factor 2 (ATF-2) but the in vivo relevance of this phosphorylation is not yet clear. ${ }^{21}$ Elk-1, which activates c-Fos, is another transcription protein that is phosphorylated by ERK1/2.

"ERK1/2 as well as other MAP kinases target not only transcription factors but also membrane proteins"

Although the primary role of ERK1/2 mediated signalling has long been thought to be restricted to cell growth and proliferation, it has become clear that several inflammatory processes involve ERKl/2 activation. Erk-1 deficient mice were normal and fertile but showed defective thymocyte maturation and reduced expression of $\alpha$ and $\beta$ chains of the T cell receptor. This indicates that ERK activation is crucial for $\mathrm{T}$ cell activation, and this was reported to be mediated by the AP-1 family of transcription factors.

\section{The JNK signal transduction pathway}

The JNK signalling pathway is a cell stress activated pathway that is involved in the regulation of cell proliferation and apoptosis. JNK protein kinases are encoded by the three genes JNK-1, JNK-2, and JNK-3, which are alternatively spliced to form the JNK isoforms (table 1.). ${ }^{22}$ In parallel with ERKI/2, JNK appears to be essential for AP-1 activation caused by stress and exposure to various cytokines. ${ }^{23}$ The two MAPKK proteins that act as upstream JNK activators are MKK7, which is primarily activated by cytokines (TNF, IL-1), and MKK4, primarily activated by environmental stress (fig 3). MAPKKK activators of JNK are composed of a large group of more than 12 intracellular proteins, including those of the MEKK group (MEKK 1-4), although it is not clear which are relevant to specific physiological stimuli. ${ }^{24}$ Duration and amplitude of JNK activation represents the balance between external stimuli and intracellular inactivation mechanisms. Four negative JNK regulatory factors have now been identified: MAP kinase phosphatase MKP7, heat shock protein 72, Evil oncoprotein, and nitric oxide. ${ }^{24}$ The two primary AP- 1 transcription components that are phosphorylated by JNK are c-Jun and ATF-2.

\section{"The JNK signalling pathway is a cell stress activated pathway that is involved in the regulation of cell proliferation and apoptosis"}

The three genes JNKl-3 have been disrupted in mice; this results in defects in immune responses and apoptosis. Isolated embryonic fibroblasts demonstrated defective AP-1 transcription activity, decreased proliferation, and resistance to stress induced apoptosis. ${ }^{25}$ Gene knockout studies targeting MKK7 
showed that TNF activates JNK through MKK7 whereas basal activity of MKK4 is required for full activation of JNK in response to TNF. ${ }^{26} \mathrm{JNK} 2$ was shown to have a crucial role in Th cell differentiation and cytokine production. ${ }^{27} \mathrm{JNK} 2^{---}$Th2 cells produced markedly reduced amounts of IFN- $\gamma$, a key cytokine for Thl cells. The JNK signalling pathway has been implicated in a large variety of pathological conditions, including cancer, stroke, ischaemic heart disease, and inflammatory disorders, and has therefore been appreciated as an attractive candidate for drug development.

\section{The p38 MAP kinase signal transduction pathway}

The p38 MAP kinase pathway shares many similarities with the other MAP kinase cascades, being associated with inflammation, cell growth, cell differentiation, and cell death. Murine p38 was first identified in 1994 as a kinase activated in response to bacterial lipopolysaccharide (LPS).$^{28}$ To date, four p38 MAP kinase isoforms have been identified sharing about $60 \%$ homology (table 1), and two isoforms (p38 $\alpha, \mathrm{p} 38 \beta$ ) are ubiquitously expressed. ${ }^{29} \mathrm{p} 38 \gamma$ is predominantly expressed in skeletal muscle whereas $\mathrm{p} 38 \delta$ gene expression is found in the lungs, kidneys, testis, pancreas, and small intestine. In most inflammatory cells, p38 $\alpha$ is the major isoform that is activated. Extracellular stimuli of the p38 MAP kinase pathway include a variety of cytokines (IL-1, IL-2, IL-7, IL-17, IL-18, TGF- $\beta$, and TNF- $\alpha$ ) and a number of pathogens that activate p38 through the different Toll receptors, including LPS, staphylococcal peptidoglycan, staphylococcal enterotoxin $\mathrm{B}$, echovirus 1 , and herpes simplex virus $1 .{ }^{29}$ Moreover, several growth factors (that is, granulocyte macrophage-colony stimulating factor, colony stimulating factor 1 , erythropoietin) $)^{30}$ are capable of inducing p38 as well as environmental factors such as heat shock, changes in osmolarity, ultraviolet, oxygen radicals, and hypoxic states. ${ }^{29}$

"p38 MAP kinase probably plays a central role in the regulation of a wide range of immunological responses"

MKK3, MKK4, and MKK6 serve as upstream MAPK kinases responsible for p38 activation, although MKK3 activates mainly $\mathrm{p} 38 \alpha, \mathrm{p} 38 \gamma$, and p38 $\delta$ (fig 3 ). A large group of proteins, acting as MAPKK kinases, activate the MKK/p38 pathway, which explains why this cascade can be activated by such a variety of stimuli. Downregulation of $\mathrm{p} 38$ activation is achieved by specific phosphatases capable of dephosphorylating not only activated p38 MAP kinases but also ERK and $\mathrm{JNK}^{31}$ The downstream targets of $\mathrm{p} 38$ are either other kinases or transcription factors such as ATF-2 and MEF2. ATF-2 can form heterodimers with Jun transcription factors and thus associates with AP-1. p38 has also been associated with activation of NFKB, as a p38 inhibitor (SB203580) has been shown to attenuate NFKB dependent transcription. ${ }^{32}{ }^{33}$ MAPKAP kinase 2 (MK2) is one of several kinases that are regulated through direct phosphorylation by p38 MAP kinase. Targeting the MK2 gene, it has been reported that mice lacking MK2 show increased stress resistance and survive LPS induced endotoxic shock due to a reduction in the production of TNF- $\alpha$ by approximately $90 \%{ }^{34}$ Thus this seems to be a posttranscriptional event as TNF- $\alpha$ mRNA is not reduced and TNF- $\alpha$ secretion is not affected.

The main biological response of $\mathrm{p} 38$ activation involves the production and activation of inflammatory mediators to initiate leucocyte recruitment and activation. For example, TNF- $\alpha$ induced upregulation of E-selectin, expressed on the surface of endothelial cells during selectin mediated rolling of leucocytes, is partly regulated through $\mathrm{p} 38 .{ }^{35}$ Furthermore, it was demonstrated that LPS stimulation of neutrophils resulted in p38 activation, leading to cell adhesion and regulation of TNF- $\alpha$ synthesis. ${ }^{33}$ Also, p38 can regulate TNF- $\alpha$ induced expression of vascular cell adhesion molecule 1 in endothelial cells, ${ }^{36}$ and chemoattractants such as $\mathrm{N}$-formyl-methionylleucyl-phenylalanine, platelet activating factor, and TGF- $\beta$ can induce neutrophil chemotaxis through p38 activation. ${ }^{37}$ Thus p38 MAP kinase probably plays a central role in the regulation of a wide range of immunological responses, as seen in inflammatory disorders, but there is a considerable overlap with other signalling routes such as ERK and JNK. In inflammatory bowel disease, it was recently reported that p38 $\alpha$ expression was increased in gut lamina propria macrophages and neutrophils. ${ }^{39}$

\section{CROSSTALK BETWEEN MAP KINASES}

Although MAP kinases generally function as autonomous signalling modules, crosstalk between the different pathways exist. This crosstalk localises to two different levels: upstream and downstream activators. The most prominent example is GTPase p21Rac that is activated by a variety of proinflammatory mediators, in particular colony stimulating factor 1 and TNF- $\alpha{ }^{40}$ In turn, Rac activates the MEK kinase p65PAK, at least in myeloid and lymphoid cells, ${ }^{41}$ which in turn activates MKK3, 4, 6, and 7, and thus is responsible for simultaneous activation of the $\mathrm{p} 38$ MAP kinase and JNK pathways, a general feature observed in most proinflammatory reactions. ${ }^{42}{ }^{43}$ Also, downstream of MAP kinases scope exists for crosstalk. Activation of MAP kinases causes activation of so-called dual specificity phosphatases, dephosphorylating MAP kinases and thus responsible for the temporal limitations in MAP kinase signalling. As the specificity of these phosphatases is limited, activation of one MAP kinase will result in the deactivation of other MAP kinases. Thus crosstalk can result in both cooperative interaction (activation of one MAP kinase leading to activation of another isoform) as well as lateral inhibition (inhibition of MAP kinases by isoforms). The actual outcome of these interactions is of course highly dependent on the cellular context.

\section{TARGETING MAP KINASE PATHWAYS FOR ANTI-INFLAMMATORY DRUG DEVELOPMENT}

The MAP kinase signalling pathways are new hunting grounds for pharmaceutical companies. Developing drugs that interfere with signalling routes and gene expression, aiming at attenuating the proinflammatory response, could prove to be a promising avenue. Most of the protein kinase inhibitors are small molecules that either interfere with phosphorylation or bind (competitive) in the ATP binding site, an area within the activation loop of the MAP kinase in which the dual phosphorylation takes place.

\section{Inhibition of MEK1/2}

Although no data are available on the use of MEK inhibitors in inflammatory models, we will discuss several compounds briefly. Two companies have developed inhibitors of MEK1/2 which are commercially available and are currently being developed as therapeutic agents against cancer (fig 4) ${ }^{44-46}$ Both inhibitors, PD98059 and U0126, appear to block MEK1 phosphorylation and subsequent activation of ERK1/2. A second generation MEK inhibitor, PD184352, has recently been shown to inhibit elevated ERK activity in colon carcinoma cells, thereby inhibiting tumour growth, ${ }^{47}$ and is now being tested in phase I clinical oncology trials.

"Two companies have developed inhibitors of MEK 1/2 which are commercially available and are currently being developed as therapeutic agents against cancer"

Roche identified a selective in vitro MEKl inhibitor (Ro 092210) in fermented microbial extracts, which was able to block anti-CD3 induced peripheral blood T cell activation and 


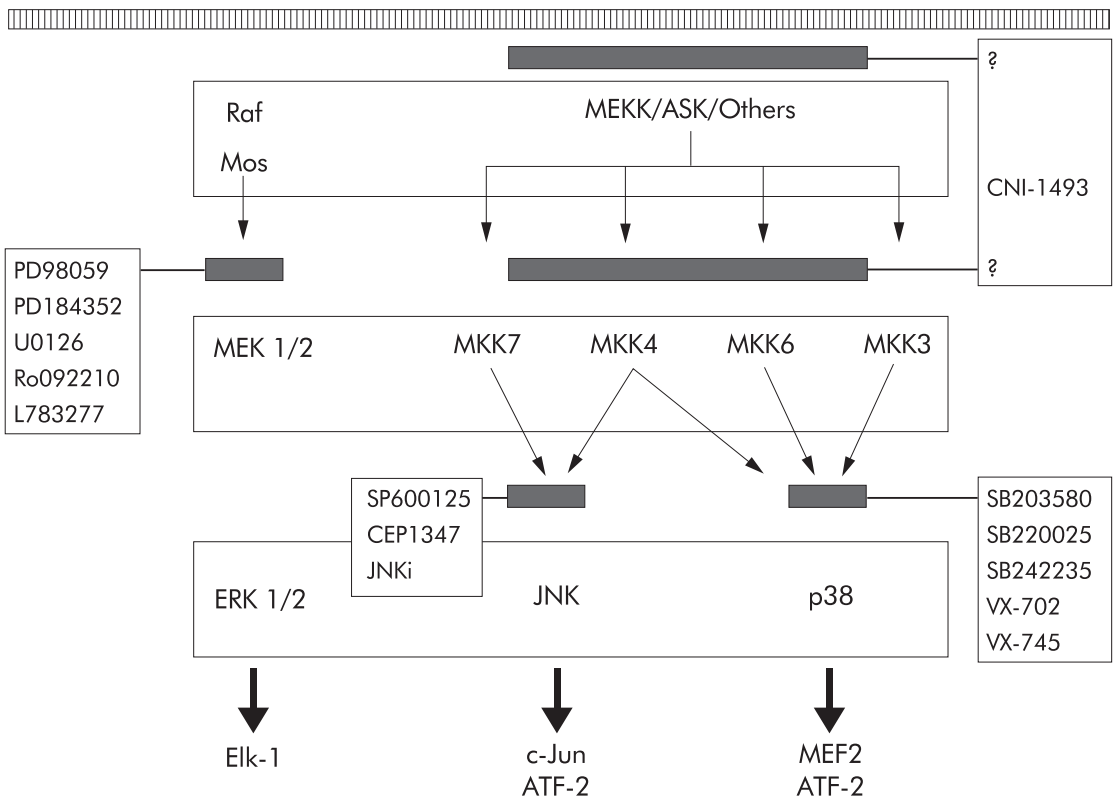

Figure 4 Different levels of pharmacological modulation of the mitogen activated protein (MAP) kinase signal transduction pathway. ATF-2, activating transcription factor 2; ERK, extracellular signal regulated kinase; JNK, c-Jun $\mathrm{NH}_{2}$ terminal kinase; MEF, myocyte enhance factor; MEK, MAP/ERK kinase; MKK, MAP kinase kinase; ASK, apoptosis signal regulating kinase.

antigen induced IL-2 secretion. ${ }^{48}$ Recently, Merck reported that a resorcylic acid lactone, L783277, also isolated from microbial extracts, inhibits MEK effectively in vitro. ${ }^{49}$ In models of traumatic brain injury, MEK inhibitors were beneficial..$^{50}$ In a rat model of global brain ischaemic tolerance, inhibition of ERK activation after preconditioning ischaemia by PD98059 significantly prevented the inhibitory effects of preconditioning ischaemia on ischaemic injury ${ }^{51}$ and reduced inflammatory responses to Helicobacter pylori LPS in rats. ${ }^{52}$ Thus it should be interesting to observe the effects of such inhibitors in human disease.

\section{Inhibition of JNK}

No specific JNK inhibitor has entered clinical evaluation. SP600125, developed by Celgene, is an inhibitor of JNK2 that does not interfere with the ERK or p38 MAP kinase pathway and reduces paw swelling in a rat model of inflammatory arthritis. ${ }^{53}$ CEP1347, a compound that inhibits members of the so-called mixed lineage kinases which are upstream activators of the JNK pathway, is a potent anti-inflammatory compound in experimental arthritis. ${ }^{54}$ Moreover, in acute experimental cerulein pancreatitis, treatment with CEP1347 resulted in significant amelioration and reduction of pancreatic edema. ${ }^{55} \mathrm{~A}$ membrane permeable peptide inhibitor of JNK (JNKi) has not been tested in vivo but inhibits AP-1 activation in alveolar macrophages. Thus pharmacological JNK inhibition has important anti-inflammatory consequences and emerges as a promising approach for combatting inflammatory disease in human pathology.

\section{Inhibition of p38 MAP kinase}

Signalling through p38 is required for synthesis of several proinflammatory cytokines, and several more or less selective p38 inhibitors have been developed (fig 4). Elucidation of the structural aspects of inhibitor/kinase interaction by $x$ ray crystallography, mutagenesis, and mechanistic enzymology has improved the potency and selectivity of these compounds. The anti-inflammatory agent pyridinylimidazole and its analogues (SB (SmithKline Beecham) compounds) were shown to sup- press proinflammatory cytokines through inhibition of $\mathrm{p} 38 \alpha$ and p $38 \beta$ in several animal models of inflammation. ${ }^{56}$ In vitro studies as well as models of gene disruption demonstrated that SB203580, a widely used p38 inhibitor, can regulate gene transcription of IL- 12 and IFN- $\gamma$ and thus also the Thl type immune responses. ${ }^{57} 58$ In a murine model of endotoxin shock, SB203580 reduced mortality. Furthermore, SB203580 showed beneficial effects in collagen induced arthritis in mice. ${ }^{56}$ In TNBS induced colitis, SB203580 did not ameliorate inflammation but suppressed the proinflammatory cytokines IL- 12 and IFN- $\boldsymbol{\gamma}^{59}$

A potential anti-inflammatory drug is CNI-1493, a synthetic guanylhydrazone which inhibits the phosphorylation of both p38 MAP kinase and JNK. $^{60}{ }^{61}$ CNI-1493 can suppress macrophage activation and the production of several proinflammatory cytokines, including TNF- $\alpha$, IL-1, IL-6, macrophage inflammatory protein $1 \alpha$, and macrophage inflammatory protein $1 \beta .^{62}{ }^{63}$ In animal models of endotoxic shock, ${ }^{62}$ adult respiratory distress syndrome, ${ }^{64}$ pancreatitis, ${ }^{65}$ and rheumatoid arthritis ${ }^{66}$ CNI-1493 showed protective effects. In a pilot study in Crohn's disease patients, CNI-1493 administration resulted in a significant decrease in Crohn's disease activity index as well as mucosal healing. ${ }^{67}$ This study suggests that JNK is the more relevant target for CNI-1493 treatment as JNK phosphorylation was inhibited more potently than p38 in LPS stimulated peripheral blood mononuclear cells in vitro, and in mucosal inflammatory cells in vivo. This hypothesis was further supported by recent findings in TNBS induced colitis in mice which revealed that although the p38 MAPK inhibitor SB20358 effectively inhibited p38 MAPK enzymatic activity in these mice, no attenuation of disease progression was observed. ${ }^{59}$ A large controlled dose finding multicentre study with CNI-1493 is underway.

"In a pilot study in Crohn's disease patients, CNI-1493 administration resulted in a significant decrease in Crohn's disease activity index as well as mucosal healing" 
In rheumatoid arthritis, all three MAP kinases are expressed in rheumatoid synovial tissues, and p38 was targeted for the development of several oral inhibitors: SB220025 was tested in a murine model of collagen induced arthritis and prevented progression of disease. ${ }^{68}$ SB242235, another member of the pyridinyl imidazole class, potently inhibits TNF- $\alpha$ and showed protective effects in antigen induced arthritis. ${ }^{69}$ Finally, two recently developed compounds VX-745 and VX-702 are now under clinical investigation for rheumatoid arthritis.

\section{TARGETING MAP KINASES FOR THE DEVELOPMENT OF ANTI-ONCOGENIC DRUGS}

Various MAP kinase pathways have been linked to processes relevant for oncogenesis. More specifically, ERK activation is considered essential for entry into the cell cycle and thus mitogenesis. Inhibition of ERK may therefore be useful in inhibiting tumour growth. Activation of the JNK pathway is associated in various ways with the apoptosis processes, and modulators of the JNK pathway may become useful for induction of cancer cell death or sensitising cancer cells to apoptosis on radiation therapy or chemotherapy. p38 MAP kinase, with its obvious role in inflammation, is often considered as a target with respect to leukaemia. Nevertheless, in vivo data as to the efficacy of such inhibitors in patients or even in animal models of oncogenic disease are still relatively sparse. No in vivo data, either in humans or in rodent models of oncogeneic disease, have as yet been reported with respect to either the JNK or p38 MAP kinase pathway. An important study by Sebolt-Leopold et al described the discovery of an orally active MEK inhibitor with substantial in vivo bioactivity in mice with colon carcinomas of both mouse and human origin. ${ }^{47}$ Thus MEK inhibitors are currently undergoing clinical trails but not much data have, as yet, entered the public domain. Various inhibitors of Raf, which act upstream of MEK, have also been shown to reduce tumour growth in xenograft models encompassing tumours of the colon, lung, and pancreas but human data are still not available. ${ }^{70}$ The clinical effects of farnesyl transferase inhibitors, which target different variants of the even more upstream Ras proteins, have until now been somewhat disappointing. ${ }^{71}$ Hence the actual usefulness of targeting MAP kinase pathways for combatting human cancer remains unproved.

\section{CONCLUSIONS}

Increased understanding of signal transduction mechanisms and gene regulation involved in immune responses has created opportunities for the discovery of novel therapeutic compounds useful in treating inflammatory disorders. These new "design drugs" seem attractive because they are capable not only of reducing a spectrum of proinflammatory cytokines but many are small molecules that can be administered orally. Although MAP kinases are necessary for life, the reported clinical side effects of MAP kinase inhibitors have not precluded further clinical development. Moreover, MAP kinase inhibitors have been used in combination with other immunosuppressive drugs, including biological therapy. Finally, production of oral MAP kinase inhibitors will not be as costly as, for example, antibody based therapies.

\section{Authors' affiliations}

D W Hommes, S J H van Deventer, Department of Gastroenterology and Hepatology, Academic Medical Centre, Amsterdam, the Netherlands

M P Peppelenbosch, Department of Experimental Internal Medicine, Academic Medical Centre, Amsterdam, the Netherlands

\section{REFERENCES}

1 Brivanlou AH, Darnell JE Jr. Signal transduction and the control of gene expression. Science 2002;295:813-18.
2 Dong C, Davis RJ, Flavell RA. Map kinases in the immune response. Annu Rev Immunol 2002;20:55-72.

3 Hunter T. Signaling - 2000 and beyond. Cell 2000;100:113-27.

4 Massague J. How cells read TGF-beta signals. Nat Rev Mol Cell Biol 2000; 1: 169-78

5 Darnell JE Jr. STATs and gene regulation. Science 1997;277:1630-5.

6 Perkins ND. The Rel/NF-kappa B family: friend and foe. Trends Biochem Sci 2000;25:434-40.

7 Barth Al, Pollack AL, Altschuler $\mathrm{Y}$, et al. $\mathrm{NH}$ 2-terminal deletion of beta-catenin results in stable colocalization of mutant beta-catenin with adenomatous polyposis coli protein and altered MDCK cell adhesion. J Cell Biol 1997; 136:693-706.

8 Allman D, Punt JA, Izon DJ, et al. An invitation to T and more: notch signaling in lymphopoiesis. Cell 2002;109(suppl):S1-1 1

9 Izon DJ, Punt JA, Pear WS. Deciphering the role of Notch signaling in lymphopoiesis. Curr Opin Immunol 2002;14:192-9.

10 Justice NJ, Jan YN. Variations on the Notch pathway in neural development. Curr Opin Neurobiol 2002; 12:64-70.

11 Crabtree GR, Olson EN. NFAT signaling: choreographing the social lives of cells. Cell 2002;109(suppl):S67-79.

12 Ikeda A, Nishina PM, Naggert JK. The tubby-like proteins, a family with roles in neuronal development and function. J Cell Sci 2002;115:9-14.

13 Kyriakis JM, Avruch J. Mammalian mitogen-activated protein kinase signal transduction pathways activated by stress and inflammation. Physiol Rev 2001;81:807-69.

14 Lewis TS, Shapiro PS, Ahn NG. Signal transduction through MAP kinase cascades. Adv Cancer Res 1998:74:49-139.

15 Fukunaga $\mathbf{R}$, Hunter T. MNK1, a new MAP kinase-activated protein kinase, isolated by a novel expression screening method for identifying protein kinase substrates. EMBO J 1997;16:1921-33.

16 Waskiewicz AJ, Flynn A, Proud CG, et al. Mitogen-activated protein kinases activate the serine/threonine kinases Mnkl and Mnk2. EMBO J 1997; 16:1909-20.

17 Boulton TG, Yancopoulos GD, Gregory JS, et al. An insulin-stimulated protein kinase similar to yeast kinases involved in cell cycle control. Science 1990;249:64-7.

18 Boulton TG, Nye SH, Robbins DJ, et al. ERKs: a family of protein-serine/threonine kinases that are activated and tyrosine phosphorylated in response to insulin and NGF. Cell 1991;65:663-75.

19 Todd JL, Tanner KG, Denu JM. Extracellular regulated kinases (ERK) 1 and ERK2 are authentic substrates for the dual-specificity protein-tyrosine phosphatase VHR. A novel role in down-regulating the ERK pathway. J Biol Chem 1999;274:13271-80

20 Pearson G, Robinson F, Beers GT, et al. Mitogen-activated protein (MAP) kinase pathways: regulation and physiological functions. Endocr Rev 2001;22:153-83.

21 Gupta S, Campbell D, Derijard B, et al. Transcription factor ATF2 regulation by the JNK signal transduction pathway. Science 1995;267:389-93.

22 Gupta S, Barrett T, Whitmarsh AJ, et al. Selective interaction of JNK protein kinase isoforms with transcription factors. EMBO J 1996; 15:2760-70

23 Ip YT, Davis RJ. Signal transduction by the c-Jun N-terminal kinase (JNK) - from inflammation to development. Curr Opin Cell Biol 1998; 10:205-19.

24 Weston CR, Davis R. The JNK signal transduction pathway. Curr Opin Genet Dev 2002;12:14-21.

25 Tournier C, Hess P, Yang DD, et al. Requirement of JNK for stress-induced activation of the cytochrome c- mediated death pathway. Science 2000;288:870-4.

26 Tournier C, Dong C, Turner TK, et al. MKK7 is an essential component of the JNK signal transduction pathway activated by proinflammatory cytokines. Genes Dev 2001;15:1419-26.

27 Yang DD, Conze D, Whitmarsh AN, et al. Differentiation of CD4+ T cells to Th 1 cells requires MAP kinase JNK2. Immunity 1998;9:575-85.

28 Han J, Lee JD, Bibbs L, et al. A MAP kinase targeted by endotoxin and hyperosmolarity in mammalian cells. Science 1994;265:808-11.

29 Ono K, Han J. The p38 signal transduction pathway: activation and function. Cell Signal 2000;12:1-13.

30 Foltz IN, Lee JC, Young PR, et al. Hemopoietic growth factors with the exception of interleukin-4 activate the p38 mitogen-activated protein kinase pathway. J Biol Chem 1997;272:3296-301.

31 Sun H, Charles CH, Lau LF, et al. MKP-1 (3CH134), an immediate early gene product, is a dual specificity phosphatase that dephosphorylates MAP kinase in vivo. Cell 1993;75:487-93.

32 Bergmann $M$, Hart L, Lindsay $M$, et al. IkappaBalpha degradation and nuclear factor-kappaB DNA binding are insufficient for interleukin-1 beta and tumor necrosis factor-alpha- induced kappaB-dependent transcription. Requirement for an additional activation pathway. J Biol Chem 1998;273:6607-10.

33 Nick JA, Avdi NJ, Young SK, et al. Selective activation and functional significance of p38alpha mitogen-activated protein kinase in lipopolysaccharide-stimulated neutrophils. J Clin Invest 1999;103:851-8.

34 Kotlyarov A, Neininger A, Schubert C, et al. MAPKAP kinase 2 is essential for LPS-induced TNF-alpha biosynthesis. Nat Cell Biol 1999:1:94-7

35 Read MA, Whitley MZ, Gupta S, et al. Tumor necrosis factor alpha-induced E-selectin expression is activated by the nuclear factor-kappaB and c-JUN N-terminal kinase/p38 mitogen-activated protein kinase pathways. J Biol Chem 1997;272:2753-61.

36 Pietersma A, Tilly BC, Gaestel $M$, et al. p38 mitogen activated protein kinase regulates endothelial VCAM-1 expression at the 
post-transcriptional level. Biochem Biophys Res Commun 1997;230:44-8

37 Hannigan $M$, Zhan $L, A i Y$, et al. The role of p38 MAP kinase in TGF-beta 1-induced signal transduction in human neutrophils. Biochem Biophys Res Commun 1998:246:55-8.

38 Zu YL, Qi J, Gilchrist A, et al. p38 mitogen-activated protein kinase activation is required for human neutrophil function triggered by TNF-alpha or FMLP stimulation. J Immunol 1998;160:1982-9.

39 Waetzig GH, Seegert D, Rosenstiel P, et al. p38 mitogen-activated protein kinase is activated and linked to TNF-alpha signaling in inflammatory bowel disease. J Immunol 2002;168:5342-51.

40 Peppelenbosch $M$, Boone $E$, Jones GE, et al. Multiple signal transduction pathways regulate TNF-induced actin reorganization in macrophages: inhibition of $\mathrm{Cdc} 42$-mediated filopodium formation by TNF. J Immunol 1999;162:837-45.

41 Blume-Jensen P, Hunter T. Oncogenic kinase signalling. Nature 2001;411:355-65

42 Coso OA, Chiariello M, Yu JC, et al. The small GTP-binding proteins Racl and Cdc42 regulate the activity of the JNK/SAPK signaling pathway. Cell 1995:81:1137-46.

43 Minden A, Lin A, Claret FX, et al. Selective activation of the JNK signaling cascade and c-Jun transcriptional activity by the small GTPases Rac and Cdc42Hs. Cell 1995;81:1 147-57.

44 Alessi DR, Cuenda A, Cohen P, et al. PD 098059 is a specific inhibitor of the activation of mitogen-activated protein kinase kinase in vitro and in vivo. J Biol Chem 1995;270:27489-94.

45 Dudley DT, Pang L, Decker SJ, et al. A synthetic inhibitor of the mitogen-activated protein kinase cascade. Proc Natl Acad Sci U S A $1995: 92: 7686-9$

46 Favata MF, Horiuchi KY, Manos EJ, et al. Identification of a novel inhibitor of mitogen-activated protein kinase kinase. J Biol Chem 1998;273: 18623-32.

47 Sebolt-Leopold JS, Dudley DT, Herrera R, et al. Blockade of the MAP kinase pathway suppresses growth of colon tumors in vivo. Nat Med 1999:5:810-16.

48 Williams DH, Wilkinson SE, Purton T, et al. Ro 09-2210 exhibits potent anti-proliferative effects on activated T cells by selectively blocking MKK activity. Biochemistry 1998;37:9579-85.

49 Zhao A, Lee SH, Mojena M, et al. Resorcylic acid lactones: naturally occurring potent and selective inhibitors of MEK. J Antibiot (Tokyo) 1999;52:1086-94

50 Mori T, Wang X, Jung JC, et al. Mitogen-activated protein kinase inhibition in traumatic brain injury: in vitro and in vivo effects. J Cereb Blood Flow Metab 2002;22:444-52.

51 Gu Z, Jiang Q, Zhang G. Extracellular signal-regulated kinase and c-Jun $\mathrm{N}$-terminal protein kinase in ischemic tolerance. Neuroreport $2001 ; 12: 3487-91$

52 Slomiany BL, Slomiany A. Role of ERK and p38 mitogen-activated protein kinase cascades in gastric mucosal inflammatory responses to Helicobacter pylori lipopolysaccharide. IUBMB Life 200 1;51:315-20.

53 Han Z, Boyle DL, Chang L, et al. c-Jun N-terminal kinase is required for metalloproteinase expression and joint destruction in inflammatory arthritis. J Clin Invest 2001;108:73-81.

54 Maroney AC, Finn JP, Connors TJ, et al. Cep-1347 (KT7515), a semisynthetic inhibitor of the mixed lineage kinase family. J Biol Chem 2001;276:25302-8.
55 Fleischer F, Dabew R, Goke B, et al. Stress kinase inhibition modulates acute experimental pancreatitis. World J Gastroenterol $2001 ; 7: 259-65$.

56 Badger AM, Bradbeer JN, Votta B, et al. Pharmacological profile of SB 203580, a selective inhibitor of cytokine suppressive binding protein/p38 kinase, in animal models of arthritis, bone resorption, endotoxin shock and immune function. J Pharmacol Exp Ther 1996;279: 1453-61.

57 Cuenda A, Rouse J, Doza YN, et al. SB 203580 is a specific inhibitor of a MAP kinase homologue which is stimulated by cellular stresses and interleukin-1. FEBS Lett 1995;364:229-33.

58 Kumar S, Jiang MS, Adams JL, et al. Pyridinylimidazole compound SB 203580 inhibits the activity but not the activation of p38 mitogen-activated protein kinase. Biochem Biophys Res Commun 1999;263:825-31.

59 ten Hove T, van Den BB, Pronk I, et al. Dichotomal role of inhibition of p38 MAPK with SB 203580 in experimental colitis. Gut 2002; 50:507-12.

60 Cohen PS, Nakshatri H, Dennis J, et al. CNI-1493 inhibits monocyte/macrophage tumor necrosis factor by suppression of translation efficiency. Proc Natl Acad Sci USA 1996:93:3967-71.

61 Cohen PS, Schmidtmayerova H, Dennis J, et al. The critical role of p38 MAP kinase in T cell HIV-1 replication. Mol Med 1997;3:339-46.

62 Bianchi $M$, Ulrich $\mathrm{P}$, Bloom $\mathrm{O}$, et al. An inhibitor of macrophage arginine transport and nitric oxide production (CNI-1493) prevents acute inflammation and endotoxin lethality. Mol Med 1995;1:254-66.

63 Bianchi M, Bloom O, Raabe T, et al. Suppression of proinflammatory cytokines in monocytes by a tetravalent guanylhydrazone. J Exp Med 1996; 183:927-36.

64 Denham W, Yang J, Wang $\mathrm{H}$, et al. Inhibition of $\mathrm{p} 38$ mitogen activate kinase attenuates the severity of pancreatitis-induced adult respiratory distress syndrome. Crit Care Med 2000;28:2567-72.

65 Yang J, Denham W, Tracey KJ, et al. The physiologic consequences of macrophage pacification during severe acute pancreatitis. Shock 1998;10:169-75

66 Akerlund K, Erlandsson HH, Tracey KJ, et al. Anti-inflammatory effects of a new tumour necrosis factor-alpha (TNF-alpha) inhibitor (CNI-1493) in collagen-induced arthritis $(\mathrm{CIA})$ in rats. Clin Exp Immunol $1999 ; 115: 32-41$

67 Hommes D, van Den BB, Plasse T, et al. Inhibition of stress-activated MAP kinases induces clinical improvement in moderate to severe Crohn's disease. Gastroenterology 2002;122:7-14.

68 Jackson JR, Bolognese B, Hillegass L, et al. Pharmacological effects of SB 220025, a selective inhibitor of P38 mitogen-activated protein kinase, in angiogenesis and chronic inflammatory disease models. J Pharmacol Exp Ther 1998:284:687-92.

69 Badger AM, Griswold DE, Kapadia R, et al. Disease-modifying activity of SB 242235, a selective inhibitor of p38 mitogen-activated protein kinase, in rat adjuvant-induced arthritis. Arthritis Rheum 2000;43: 175-83.

70 Herrera R, Sebolt-Leopold JS. Unraveling the complexities of the Raf/MAP kinase pathway for pharmacological intervention. Trends Mol Med 2002;8:S27-31.

71 Prendergast GC, Rane N. Farnesyltransferase inhibitors: mechanism and applications. Expert Opin Investig Drugs 2001;10:2105-16. 